\title{
FR dichotomy, accretion modes and environmental factors in the Local Universe
}

\author{
M. A. Gendre* \\ Jodrell Bank Center for Astrophysics \\ E-mail: mgendreejb. man.ac.uk

\section{P. N. Best} \\ Institute for Astronomy, Royal Observatory
}

\section{J. V. Wall}

Department of Physics and Astronomy, The University of British Colombia

\begin{abstract}
The goal of this work is to determine the nature of the relation between morphology and accretion mode in radio galaxies, including environmental parameters. The CoNFIG extended catalogue, improved by including additional information such as $\mathrm{K}_{S}$-magnitudes from UKIDSS (and the related K-z estimated redshift) and spectral index derived using new GMRT observation, is used to select a morphology-classified (using the Fanaroff-Riley (FR) classification of extended radio sources) sub-sample of galaxies with $\mathrm{z} \leq 0.3$. For each source, spectroscopic data are retrieved to determine the high/low excitation status of the source related to its accretion mode. Environmental factors, such as the host galaxy luminosity and a richness factor computed using SDSS or 2MASS, are also included. We find that (1) FR morphologies do not seem to be distinguished solely based on the radio luminosity and accretion-mode of the source. This result is however greatly dependent on the errors; (2) although FRIs generally reside in denser environments that FRIIs, HEG/LEG in a poor/rich environment has roughly equal probabilities of being of morphological type-I or type-II; (3) excitation modes seem to be linked to the source environment, with HEGs found almost exclusively in low-density environments while LEGs occupy a wider range of densities.
\end{abstract}

Resolving the Sky - Radio Interferometry: Past, Present and Future -RTS2012

April 17-20, 2012

Manchester, $U K$

\footnotetext{
${ }^{*}$ Speaker.
} 


\section{The CoNFIG local sample}

In previous studies of FR sources using the CoNFIG catalogue (Gendre et al. 2008, 2010), results have shown that, at comparable powers, FRI and FRII sources show strong similarities in evolution, which indicate that they very probably share a common mechanism governing the luminosity-dependent evolution. In addition, radio luminosity function (RLF) models hint at a luminosity-dependent bimodal evolution, where low-luminosity sources show no evolution while high-luminosity sources undergo positive density evolution. What differentiates FR sources? Two main streams of models exist to explain these differences in morphology: Extrinsic models, purely based on the source environment (e.g. Prestage \& Peacock 1988) and intrinsic models, suggesting that the dichotomy arises from differences in the properties of the central black hole (e.g. Ghisellini \& Celotti 2001). To test these scenarios, high/low excitation classification and environmental richness factor of a sub-sample of local $(\mathrm{z} \leq 0.3)$ CoNFIG extended galaxies were compiled to investigate the possible FR morphology/accretion mode/environment relations. The sub-sample contains 206 sources, including 74 FRIs and 107 FRIIs, $76 \%$ of which have available spectra, mostly from SDSS.

\section{High/low excitation galaxies}

In this work, HEG/LEG classification was determined by measuring the $[\mathrm{OIII}]\left(\lambda_{[\mathrm{OIII}]}=5007 \AA\right)$ and [OII] $\left(\lambda_{[\mathrm{OII}]}=3727 \AA\right)$ lines, and following the definitions of Jackson \& Rawlings (1997): sources with rest-frame [OIII] equivalent width $<1 \mathrm{~nm}$ and/or [OII]/[OIII] $>1$ were classified as LEG, other sources being classified as HEG. If no [OIII] line was detected in the spectrum, the source was considered to be low excitation.

For the CoNFIG local sub-sample, we found 88 LEGs (including 49 FRIs and 29 FRIIs) and 70 HEGs (including 12 FRIs and 46 FRIIs). The 48 other sources (including 13 FRIs and 27 FRIIs) did not have spectra available to determine the excitation level of the host galaxy.

The local radio luminosity function was computed for both HEGs and LEGs. Looking at host galaxies properties of sources with no HEG/LEG classification available, no major systematic offset in magnitude or other properties were observed compared to other radio sources of the same redshifts and radio fluxes. These unclassified sources were therefore considered to be a random sub-sample and were taken into account by correcting each LRLFs by a factor:

$$
F=1+\frac{\left.\sum_{i=1}^{N} \frac{1}{V_{i}}\right|_{\text {unclass. }}}{\left.\sum_{i=1}^{N} \frac{1}{V_{i}}\right|_{\text {classified }}}
$$

The resulting LRLFs are shown in Figure 1. We see that for both HEGs and LEGs, the data cover the full range of radio luminosities studied $\left(22.0 \leq \log \mathrm{P}_{1.4 \mathrm{GHz}} \leq 26.0 \mathrm{~W} / \mathrm{Hz} / \mathrm{sr}\right)$, and they agree well with the work of Best \& Heckman (2012), indicating that the inclusion of sources with no HEG/LEG classification was properly done. We do find a higher space density of HEGs in our sample for $\log \mathrm{P}_{1.4 \mathrm{GHz}} \geq 24 \mathrm{~W} / \mathrm{Hz} / \mathrm{sr}$ relative to Best \& Heckman (2012), but no deficiency in LEGs. Part of this is caused by the exclusion of quasars which, although a small proportion of 


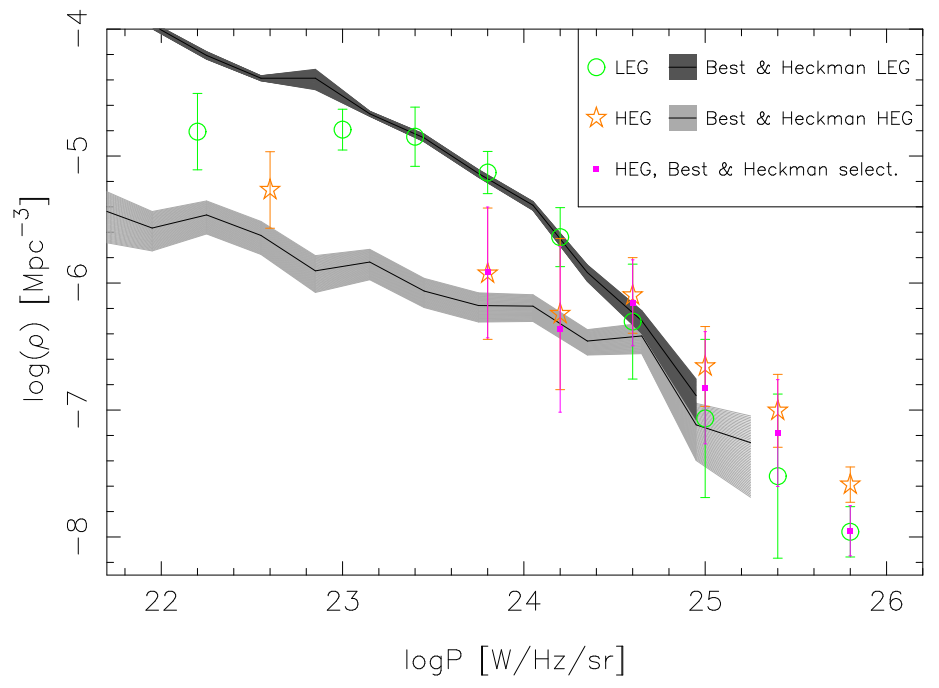

Figure 1: Local radio luminosity function $\rho(P)$ for HEGs (stars) and LEGs (circles) separately, using bin sizes of $\Delta \log \mathrm{P}_{1.4 \mathrm{GHz}}=0.4$. The LRLFs are compared to results from Best \& Heckman (2012) (in light and dark grey for HEGs and LEGs respectively). For more accurate comparisons, the LRLF for CoNFIG HEGs with SDSS counterparts (excluding QSOs) as selected by Best \& Heckman (2012) is shown in filled squares.

the overall sample, are a significant fraction of high power HEGs. Nevertheless, a small excess is still present when applying the Best \& Heckman (2012) selection criteria, suggesting that optically selected samples, such as SDSS, might be under-sampling high-power HEGs.

\section{Environmental factors}

Cluster richness for each source was determined using the method of Wing \& Blanton (2011), in which the richness factor $\mathrm{N}_{1}^{-19}$ corresponds to the corrected number of SDSS galaxies with absolute magnitudes brighter than $\mathrm{M}_{r}=-19$ within a $1.0 \mathrm{Mpc}$ radius of the radio source. The corrected galaxy count is obtained by measuring the total number of sources in the $1.0 \mathrm{Mpc}$-radius disk and subtracting a background count, measured from a shell of inner and outer radii 2.7 and $3.0 \mathrm{Mpc}$ respectively. When SDSS data were unavailable (20.4\% of the local sample), 2 MASS K -band (28 sources) and ESO Imaging Survey (EIS) Patch-D I-band (Nonino et al. 1999; 14 CENSORS sources) data were used.

According to Wing \& Blanton (2011), a cluster-richness of $\mathrm{N}_{1}^{-19} \leq 20$ likely corresponds to a poor cluster, while $\mathrm{N}_{1}^{-19} \geq 40$ corresponds to a rich cluster. It was thus decided to use $\mathrm{N}_{1}^{-19}=30$ to differentiate between poor and rich environments.

\section{Results}

\subsection{HEG/LEG}

The possibility that FR types depend on the distinct accretion mode inside the central SMBH is examined by looking at the probability of a HEG/LEG being of a given FR type. 

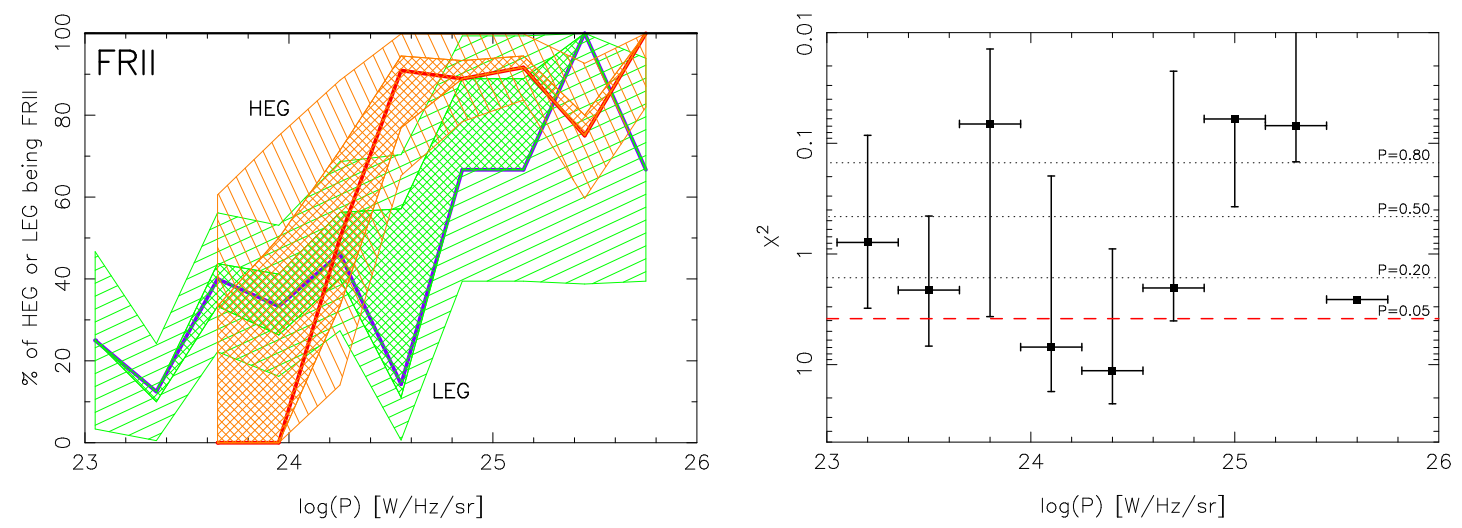

Figure 2: Left: Percentage of HEGs (thick red line) and LEGs (thick purple line) being FRII in the CoNFIG local sub-sample (excluding source with no HEG/LEG classification). The luminosity bins are $\Delta \log \mathrm{P}=0.3$. The cross-hatched regions correspond to the minimum and maximum possible values of the ratios when including sources with unidentified spectral type, and the hatched regions include errors in these limits depending on the number of sources in each bins following Poisson statistics.

Right: Result of the Pearson chi-square test performed on the FRI/FRII HEG/LEG samples for each luminosity bins. For comparison, $\chi^{2}$ values corresponding to a probability $\mathrm{P}=0.2,0.5$ and 0.8 that radio morphology is independent of excitation are displayed in dotted lines. The dashed line represent the value of $\chi^{2}$ for which $\mathrm{P}=0.05$, the lowest acceptable probability for which the distributions are independent accepted here. For each luminosity bins, the range of possible $\chi^{2}$ values when including unclassified sources is determine based on the minimum and maximum values of $\chi^{2}$ in each of the following extreme cases: (i) no unclassified sources are taken into account; (ii) all unclassified sources are LEG; (iii) all unclassified sources are HEG; (iv) all FRI unclassified sources are LEG while all FRII unclassified sources are HEG; (v) all FRI unclassified sources are HEG while all FRII unclassified sources are LEG.

The fractions of HEG and LEG being FRII are displayed in the left panel of Figure 2. The two distribution overlap within the errors, which include both uncertainty due to sources with no HEG/LEG classification (17.6\% of FRI and 26.5\% of FRIIs) and Poisson statistics dependent on the number of sources in each luminosity bin considered. It appears that Poisson errors is the main source of uncertainty here.

A Pearson chi-square test, including Yate's correction when appropriate, was performed on the FRI/FRII HEG/LEG samples for each luminosity bins (right panel of Figure 2). In most luminosity bins (aparm from $23.95 \leq \log \mathrm{P}_{1.4 \mathrm{GHz}} \leq 24.55 \mathrm{~W} / \mathrm{Hz} / \mathrm{sr}$ ), the probability of radio morphology being independent of excitation is greater than 5\%, and up to $\mathrm{P}_{F R-H / L}>80 \%$ in a third of cases. When including sources with no excitation classification, it appears possible that FRI/FRII are independent of HEG/LEG type over the whole range of luminosity considered.

Thus, based on the above results, there is a significant possibility that FR morphologies can not be distinguished solely based on the radio luminosity and accretion-mode of the source.

\subsection{Environmental influences}

\subsubsection{Cluster richness}

The idea that, no matter how they are produced, jets will behave differently depending on the 

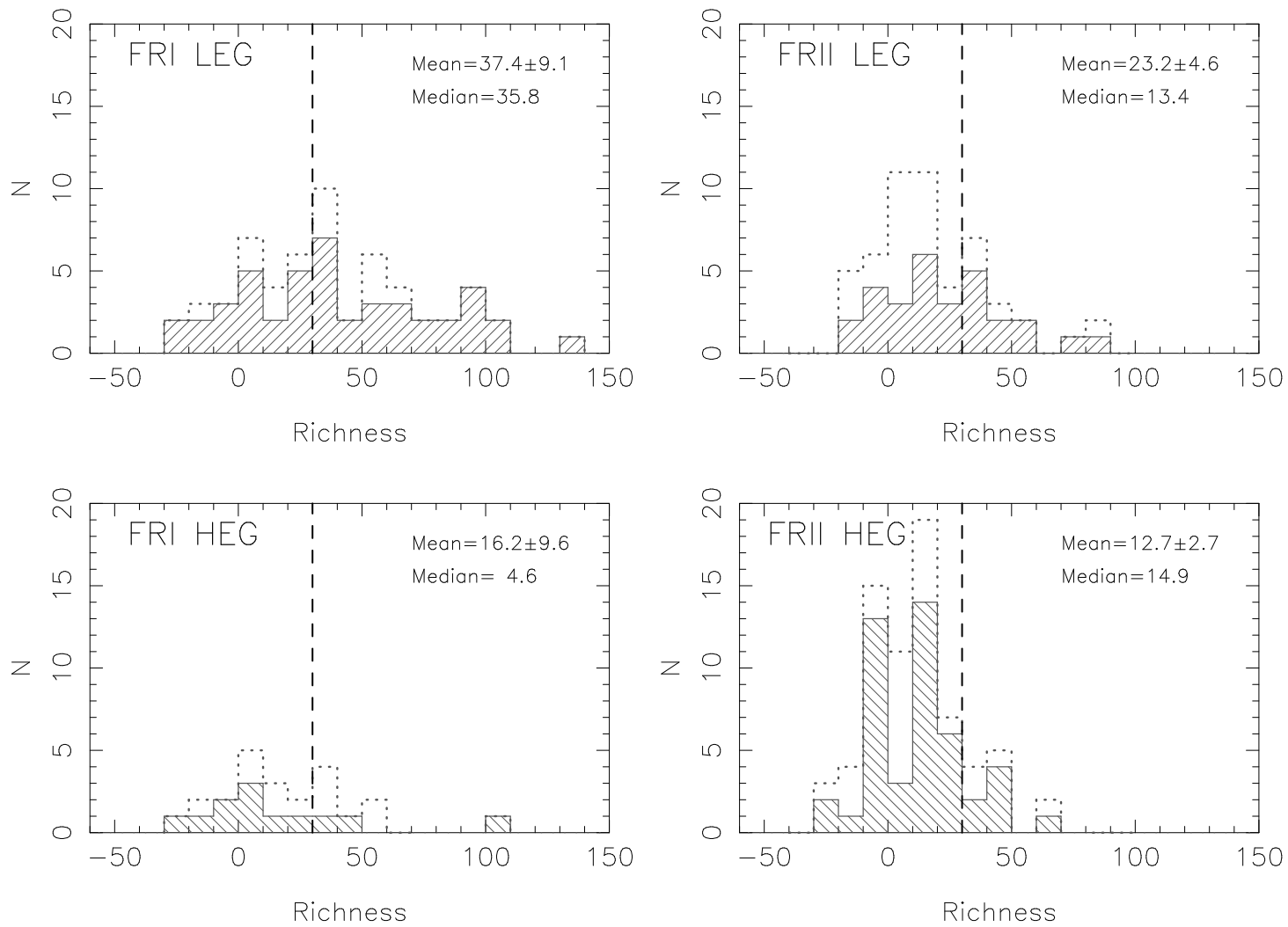

Figure 3: Richness distribution for FRI (left) and FRII (right) LEG (top) and HEG (bottom) sources in the CoNFIG local sub-sample. The dashed line correspond to $\mathrm{N}_{1}^{-19}=30$, the limit between poor and rich cluster. The richness distribution taking into account sources for which HEG/LEG classification was not possible are represented as dotted histograms. Mean and median richness (without unclassified sources) are quoted for each distribution.

cluster environment they encounter is a possible explanation for the different FR morphologies, independently of excitation types.

The richness distribution of FRI/FRII HEGs/LEGs is shown in Figure 3. To further look into a possible FR morphology - excitation mode dependence (or lack thereof), a K-S test performed for four comparative cases: FRI HEGs and LEGs, FRII HEGs and LEGs, LEG FRIs and FRIIs and HEG FRIs and FRIIs. The probabilities that the considered samples are drawn from the same distribution are $\mathrm{P}_{\mathrm{I}-\mathrm{H} / \mathrm{L}}=0.06, \mathrm{P}_{\mathrm{II}-\mathrm{H} / \mathrm{L}}=0.99, \mathrm{P}_{\mathrm{L}-\mathrm{I} / \mathrm{II}}=0.68$ and $\mathrm{P}_{\mathrm{H}-\mathrm{I} / \mathrm{II}}=0.95$ respectively. The probability $\mathrm{P}_{\mathrm{I}-\mathrm{H} / \mathrm{L}}$ seems to indicate that FRI sources are not independent of accretion mode, with a tendency to be low-excitation. However, this dependency vanishes when looking at sources with $\mathrm{N}_{1}^{-19}<30$, where the K-S test on FRI HEGs and LEGs then yielding $\mathrm{P}_{\mathrm{I}-\mathrm{H} / \mathrm{L}-\mathrm{Poor}}=0.99$. the other $\mathrm{K}-\mathrm{S}$ test results $\left(\mathrm{P}_{\mathrm{II}-\mathrm{H} / \mathrm{L}}, \mathrm{P}_{\mathrm{L}-\mathrm{I} / \mathrm{II}}\right.$ and $\left.\mathrm{P}_{\mathrm{H}-\mathrm{I} / \mathrm{II}}\right)$ suggest that radio morphology and excitation modes are independent of each other.

Looking at the richness distributions themselves, it appears that radio sources in rich clusters have a higher probability of being FRI (as previously stated by Zirbel 1997, Prestage \& Peacock 

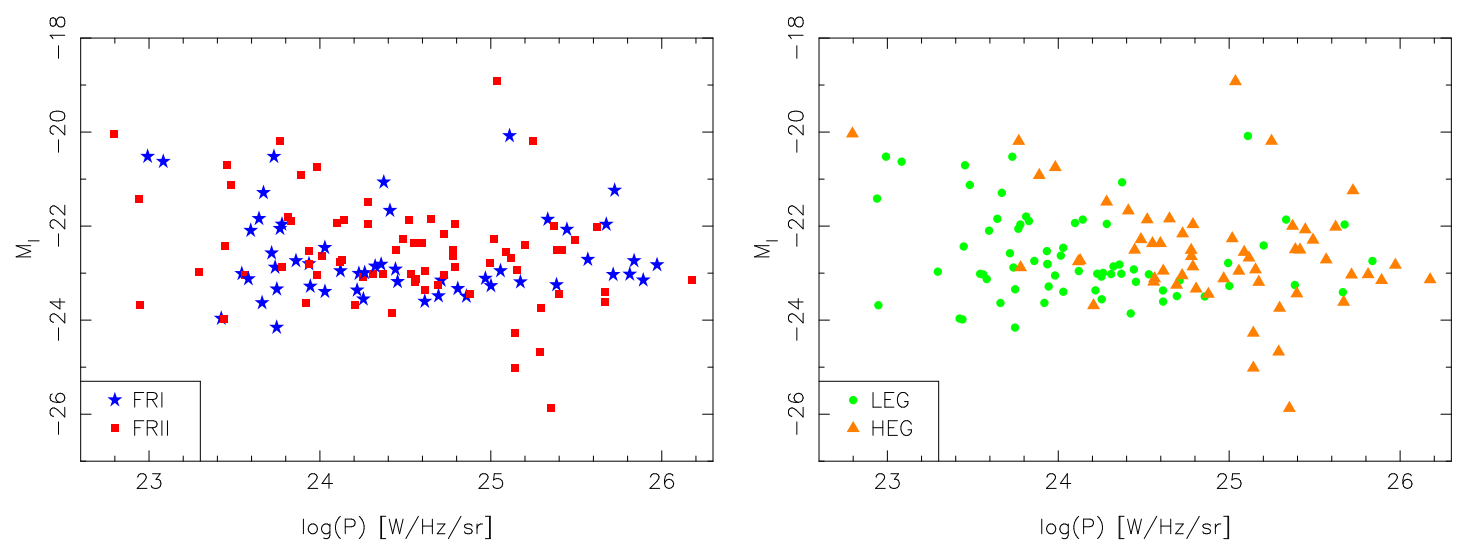

Figure 4: Optical I-band vs. radio luminosity for FRI and FRII (left panel) and LEGs and HEGs (right panel).

1988) and show low-excitation. The latter can be explained by the fact that jets in massive galaxies with low cooling-rates, giving rise to LEGs (Hardcastle et al. 2007), are easily disrupted, resulting in FRI-like morphologies in dense environments. On the other hand, a HEG/LEG in a poor/rich environment has roughly equal probabilities of being of morphological type-I or type-II, within errors.

Another interesting point from these distribution plots is that excitation modes (for both FRIs and FRIIs) seem to be linked to the source environment. Performing a Pearson chi-square test on samples of HEGs and LEGs in poor and rich clusters (ignoring unclassified sources) leads to $\chi^{2}=14.23(v=1)$, rejecting the hypothesis that excitation mode and environment parameters are independent with probability $\mathrm{P}_{H / L-R i c h}=99 \%$. The dependence of the accretion mode on the environment can possibly be explained by the feeding mechanism associated with each type. As seen in Figure 3, HEGs are found almost exclusively in low-density environments, with $\mathrm{N}_{1}^{-19}$ centered around zero, which is consistent with being the result of mergers (which tend to occur in groups, with lower densities than clusters). In contrast, LEGs are found in a wider range of densities, which is consistent with the idea that their gas supply originates from the cooling out of either the host galaxy itself (possible in both rich and poor environments) or the cluster halo (requiring high densities).

\subsubsection{Host galaxy}

According to the previous results, the disruption of the jets leading to the different FR types does not seem to happen on the large cluster scale. Another possibility considered in this work is that the disruption occurs on the scale of the host galaxy. This was suggested by Ledlow \& Owen (1996), who found that the FR division is a function of both optical and radio luminosity while considering sources in the 3CR sample. However, several recent studies (Best 2009, Wing \& Blanton 2011), based on other independent samples, failed to replicate the sharp dicision found between FR populations, finding a large overlap around the Ledlow \& Owen divide. Looking at the $\mathrm{M}_{\mathrm{I}}-\log \mathrm{P}_{1.4 \mathrm{GHz}}$ plots for the CoNFIG local sub-sample presented in Figure 4, it is apparent that the result of Ledlow \& Owen (1996) does not hold for the CoNFIG local sub-sample, even when considering the different intrinsic and extrinsic parameters. This implies that radio galaxies of 
different FR type are not hosted by significantly different galaxies. In contrast, HEGs and LEGs appear to occupy different regions of the $\mathrm{M}_{\mathrm{I}}-\log \mathrm{P}_{1.4 \mathrm{GHz}}$ space. However, this separation is mostly radio-power driven.

\section{References}

Best, P. N., 2009, A.N., 330, 184

Best, P. N. \& Heckman, T. M., 2012, MNRAS, 421, 1569

Gendre M. A. \& Wall J. V., 2008, MNRAS, 390, 819

Gendre M. A., Best, P. N. \& Wall J. V., 2010, MNRAS, 404, 1719

Ghisellini G. \& Celotti A.,2001, MNRAS, 327, 739

Hardcastle M. J., Evans D. A. \& Croston J. H., 2007, MNRAS, 376, 1849

Jackson, N. \& Rawlings, S., 1997, MNRAS, 286, 241

Janssenn, R. M. J., Röttgering, H. J. A., Best, P. N. \& Brinchmann, J., 2012, A\&A, 541, 62

Ledlow, M. J. \& Owen, F. N., 1996, AJ, 112, 9

Nonino M. et al., 1999, $A \& A S, 137,51$

Prestage R. M. \& Peacock J. A., 1988, MNRAS, 230, 131

Wing J. D. \& Blanton E. L., 2011, AJ, 141, 88

Zirbel, E. L., 1997, ApJ, 476, 489 